\title{
USAGE OF SMART CLOTHES IN MEDICAL SERVICES
}

\author{
Fotooheh teimouri
}

MS, Isfahan Health Center, Isfahan University of Medical Sciences, Isfahan, Iran

Correspondence:

F Teimouri, Tel: +98-311-4466896, Fax: +98-311-4485658, E-mail: Fotoohe@yahoo.com

\section{TYPE OF ARTICLE: CONFERENCE ABSTRACT}

\begin{abstract}
Introduction: Lack of continuous vital sign monitoring for people who need long-term health care is one of the problems in patient care. One of the new technologies in the field of health is smart clothes, which provides vital sign monitoring of patients, athletes, premature infants, children, psychiatric patients, people who need long-term care, elderly, and people in impassable regions far from health and medical services; these clothes will be significantly effective in prevention, timely diagnosis, control, and treatment of diseases. The aim of this study was to explain features and applications of smart clothes in medical services. The aim of this study was to explain the features and applications of wearable biosensors in medical services.

Methods: The study is a narrative review, which was done in 2016. A search was conducted with the help of libraries, books, conference proceedings, and databases of Science Direct, PubMed, Proquest, Springer, and SID (Scientific Information Database). In our searches, we employed the following keywords and their combinations; vital sign monitoring, medical smart shirt, smart clothing, wearable biosensors, physiological monitoring system, remote detection systems, remote control health, and bio-monitoring systems. The preliminary search resulted in 47 articles, which were published between 2002 and 2016. After careful analysis of the content of each paper, a total of 29 sources were selected based on their relevancy.

Results: According to records at home and abroad, studies have looked into the field of intelligent textiles and smart clothes, and medical studies are being conducted in the country. In this study, a set of applications has been collected. The user has a broad range of smart clothes, which are most widely used medicine for the elderly, babies, people in need long-term care, psychiatric care, rehabilitation and heart and lung disease.

Conclusion: Decreasing the volume of digital tools on the one hand and significant expansion of public access to some technologies such as mobile phones on the other have accelerated the design of smart clothing. Perhaps smart clothing was previously considered a luxury, but some applications of the clothing, particularly in the health field have converted it to the first choice in some practical areas. It is probable that, in the not too distant future, use of smart clothing will be spread as smart phones. Coming soon, smart clothes will find routine clinical applications. Recently, many companies have turned to production and development of these wearable products; undoubtedly, it can be said that the future of digital world is in the hands of wearable tools.

KEYWORDS: Smart clothes, Medical smart shirt, Physiological monitoring systems, Vital signs monitoring
\end{abstract}

\section{Abstracts of First National Congress of Medical Informatics, Mashhad, Iran, February 2017}

(C) 2017 The Authors. This is an open access article under the terms of the Creative Commons Attribution-NonCommercialNoDerivs License, which permits use and distribution in any medium, provided the original work is properly cited, the use is non-commercial and no modifications or adaptations are made. 